\title{
Clinical and laboratory characteristics in leptospirosis
}

\author{
Raluca Elena Jipa ${ }^{1 *}$, Nicoleta Andreescu², Eliza Manea', Sorinela Diaconu', Diana Niculescu', Doina Antonică', \\ Nicoleta Irimescu', Ruxandra Moroti', Adriana Hristea' ${ }^{1}$ \\ From The 10th Edition of the Scientific Days of the National Institute for Infectious Diseases "Prof Dr Matei \\ Bals" \\ Bucharest, Romania. 15-17 October 2014
}

\section{Background}

Leptospirosis is one of the most common zoonoses in the world, with a wide range of manifestations that can vary from mild to severe with acute hepatic and renal failure, pneumonia or meningitis. Between 2005-2011 in Romania, according to ECDC, 1740 cases have been reported. Objective: To describe clinical and laboratory characteristics of confirmed cases of leptospirosis.

\section{Methods}

Retrospective study, between January 2004-June 2014, in one infectious diseases hospital in Bucharest. We included patients with leptospirosis diagnosis at discharge and/or positive serology for leptospirosis. Serological diagnosis of acute leptospirosis was made by microscopic agglutination test using a battery of 17 antigens from international reference strains in one national reference laboratory and/or positive IgM-enzyme-linked immunosorbent assay (ELISA). Statistical analysis was performed with SPSS v19.0; continuous variables were described with medians and ranges; categorical variables were described with numbers and percentages.

\section{Results}

Of 132 patients with leptospirosis diagnosis at discharge, $105(80 \%)$ had positive serology for leptospirosis. The median age was 37 (IQR 29-53) and 94 (90\%) were male. 71 (68\%) patients were diagnosed between May and September, 54 (51\%) lived in urban areas and 8 (8\%) patients had professional exposure. Leptospirosis serotype has been identified in $75(71 \%)$ patients. $9(9 \%)$ patients had meningeal involvement, $12(12 \%)$ patients respiratory manifestations, $66(63 \%)$ patients renal impairment and 38 (36\%) patients coagulation impairment. The median level of alanin aminotransferase, gamma-glutamyl transpeptidase and total bilirubin were $88 \mathrm{IU} / \mathrm{mL}$ (IQR 50-158), $162 \mathrm{IU} / \mathrm{mL}$ (IQR 86-279) and $4.7 \mathrm{mg} / \mathrm{dL}$ (IQR 1.2-14.7), respectively. Leukocytosis (WBC $>10.000 / \mu \mathrm{L}$ ) was present in $55(52 \%)$ patients, severe thrombocytopenia (PLT $<50.000 / \mu \mathrm{L})$ in $25(24 \%)$ patients and $87(83 \%)$ patients had inflammatory syndrome. In-hospital mortality was $4 \%(4 / 105)$.

\section{Conclusion}

Liver and renal failure were the most common manifestations in leptospirosis. Increased awareness should be maintained in order to diagnose and initiate early adequate treatment to reduce mortality and morbidity.

\section{Authors' details}

${ }^{1}$ National Institute for Infectious Diseases "Prof. Dr. Matei Balş", Bucharest, Romania. ${ }^{2}$ Cantacuzino National Institute for Research and Development for Microbiology and Immunology, Bucharest, Romania.

Published: 15 October 2014

doi:10.1186/1471-2334-14-S7-P29

Cite this article as: Jipa et al:: Clinical and laboratory characteristics in

leptospirosis. BMC Infectious Diseases 2014 14(Suppl 7):P29.

\footnotetext{
* Correspondence: ralucajipa@yahoo.com

"National Institute for Infectious Diseases "Prof. Dr. Matei Balş", Bucharest,

Romania

Full list of author information is available at the end of the article
} 